\title{
Procedures for evaluating the HV overhead lines lightning performance and methods for reducing the OHL trip rate in TNB transmission
}

\begin{abstract}
In this paper, the procedures for evaluating the High Voltage $(132 \mathrm{kV}, 275 \mathrm{kV}$ and $500 \mathrm{kV})$ overhead lines performance due to lightning in TNB transmission is discussed. The historical tripping data is analysed and compared from the international standard IEC 60071-1 and other utility practices. Approaches for evaluating the performance i.e. software simulations using TFlash and SIGMA SLP are part of the study. Lightning stroke data will also be shown, which will be compared with the tripping pattern. The condition of the transmission towers and Tower Footing Resistance are also very essential in determining the line performance. As conclusion, this paper will discuss and propose the method and actions for reducing OHL tripping due to lightning, thus improving the line performance.
\end{abstract}

Keyword: Electric power transmission; Overhead lines; Lightning performance; HV overhead lines 